\title{
Extended-duration rivaroxaban thromboprophylaxis in acutely ill medical patients: MAGELLAN study protocol
}

\author{
Alexander Thomas Cohen - Theodore Erich Spiro • Harry Roger Büller • \\ Lloyd Haskell • Dayi Hu · Russell Hull • Alexandre Mebazaa - Geno Merli • \\ Sebastian Schellong · Alex Spyropoulos • Victor Tapson
}

Published online: 27 February 2011

(c) The Author(s) 2011. This article is published with open access at Springerlink.com

\begin{abstract}
Patients with acute medical illnesses are at increased risk of venous thromboembolism (VTE), a significant cause of morbidity and mortality. Thromboprophylaxis is recommended in these patients but questions remain regarding the optimal duration of therapy. The aim of this study is to determine whether oral rivaroxaban is non-inferior to standard-duration (approximately 10 days) subcutaneous (s.c.) enoxaparin for the prevention of VTE in acutely ill medical patients, and whether extendedduration (approximately 5 weeks) rivaroxaban is superior to standard-duration enoxaparin. Patients aged 40 years or older and hospitalized for various acute medical illnesses with risk factors for VTE randomly receive either s.c. enoxaparin $40 \mathrm{mg}$ once daily (od) for $10 \pm 4$ days or oral rivaroxaban $10 \mathrm{mg}$ od for $35 \pm 4$ days. The primary efficacy outcomes are the composite of asymptomatic proximal deep vein thrombosis (DVT), symptomatic DVT,
\end{abstract}

This study is being conducted on behalf of the MAGELLAN Steering Committee.

\author{
A. T. Cohen $(\square)$ \\ Department of Surgery and Vascular Medicine, King's College \\ Hospital, London SE5 9RS, UK \\ e-mail: alexander.cohen@kcl.ac.uk \\ T. E. Spiro \\ Bayer HealthCare Pharmaceuticals Inc., Montville, NJ, USA \\ H. R. Büller \\ Academic Medical Centre, Amsterdam, The Netherlands \\ L. Haskell \\ Johnson \& Johnson Pharmaceutical Research \& Development, \\ L.L.C, Raritan, NJ, USA \\ D. $\mathrm{Hu}$ \\ People's Hospital of Peking University, Beijing, China
}

symptomatic non-fatal pulmonary embolism (PE), and VTE-related death up to day $10+4$ and up to day $35+4$. The primary safety outcome is the composite of treatmentemergent major bleeding and clinically relevant non-major bleeding. As of July 2010, 8,101 patients from 52 countries have been randomized. These patients have a broad range of medical conditions: approximately one-third were diagnosed with acute heart failure, just under one-third were diagnosed with acute infectious disease, and just under one-quarter were diagnosed with acute respiratory insufficiency. MAGELLAN will determine the efficacy, safety, and pharmacological profile of oral rivaroxaban for the prevention of VTE in a diverse population of medically ill patients and the potential of extended-duration therapy to reduce incidence of VTE.

Keywords Anticoagulants - Enoxaparin - Rivaroxaban · Venous thromboembolism

R. Hull

Foothills Hospital, Calgary, AB, Canada

A. Mebazaa

Lariboisière University Hospital, Paris, France

G. Merli

Thomas Jefferson Medical Center, Philadelphia, PA, USA

S. Schellong

Dresden-Friedrichstadt Hospital, Dresden, Germany

A. Spyropoulos

Henderson Research Center, Hamilton, ON, Canada

V. Tapson

Duke University Medical Center, Durham, NC, USA 


\section{Introduction}

Venous thromboembolism (VTE) comprises deep vein thrombosis (DVT) and pulmonary embolism (PE). Shortterm (1-2 weeks) thromboprophylaxis with anticoagulants has resulted in a reduction of both non-fatal and fatal PE in medical and surgical patients [1-3]. Extended-duration regimens in surgical patients have successfully reduced the incidence of VTE compared with placebo [4] or the current standard of care [5-9], and their use is recommended in clinical guidelines $[4,10]$. Little is known about extendedduration regimens in medical patients.

Approximately three-quarters of all in-hospital deaths from PE occur in medical patients [11], and the prevalence of asymptomatic and symptomatic DVT in hospitalized medical patients not receiving thromboprophylaxis is between 10 and 20\% [4]. Acute medical conditions, such as acute heart failure, acute infection, acute respiratory failure, stroke, and myocardial infarction, are known to put patients at increased risk of VTE [12, 13], and most conditions that require prolonged immobilization also represent a temporary excess risk of VTE [12]. Other risk factors include age over 75 years, chronic heart failure, cancer and chemotherapy, prior VTE, obesity, varicose veins, and thrombophilia [12-16].

Large randomized clinical trials that have demonstrated the effectiveness of short-term pharmacologic thromboprophylaxis in acutely ill medical patients showed reductions in the relative risk of VTE of between 45 and 64\% [2, 17-19]. The results of these trials formed the basis of guideline recommendations for the use of unfractionated heparin, low-molecular-weight-heparins (LMWHs), or fondaparinux in this patient population [4].

Although guidelines recommend anticoagulant use in acutely ill medical patients, their use is not routine in clinical practice. International registry and epidemiological data have shown that approximately $50 \%$ of at-risk acutely ill medical patients receive any form of prophylaxis, and that less than $40 \%$ receive prophylaxis in line with recommendations from the American College of Chest Physicians (ACCP) guidelines [20, 21].

There is evidence that the risk of VTE in acutely ill medical patients persists beyond hospital discharge. In the MEDENOX study, where prophylaxis or placebo were administered for $10 \pm 4$ days, eight venous thromboembolic events ( $7.8 \%$ of the total) occurred between days 15 and 110, of which four were fatal PEs [17]. More recently, the EXCLAIM study demonstrated that extended-duration enoxaparin ( $38 \pm 4$ days), compared with placebo after open-label standard-duration enoxaparin $(10 \pm 4$ days $)$, significantly reduced the overall incidence of VTE (absolute risk difference, $-1.53 \%$ [95.8\% confidence interval [CI], -2.54 to $-0.52 \%]$ ) in acutely ill medical patients with reduced mobility, but with a significant increase in the incidence of major bleeding (absolute risk difference, $0.51 \%$ [95\% CI, 0.12 to $0.89 \%$ ]) [22].

Rivaroxaban is an oral, direct Factor Xa inhibitor approved in many countries worldwide for the prevention of VTE after elective hip and knee replacement surgery in adult patients. Rivaroxaban has a fast onset and offset of action, and has been shown to have predictable pharmacokinetics (PK) and pharmacodynamics (PD) and a low propensity for drug-drug interactions [23].

MAGELLAN (Multicenter, rAndomized, parallel Group Efficacy and safety study for the prevention of VTE in hospitalized acutely iLL medical patients comparing rivaroxabAN with enoxaparin) was designed to assess both the efficacy and safety of oral rivaroxaban compared with standard-duration subcutaneous (s.c.) enoxaparin, and to evaluate the role of extended-duration (approximately 5 weeks) thromboprophylaxis in acutely ill medical patients.

\section{Study objectives}

The objectives of the study are twofold: firstly, to determine whether oral rivaroxaban $10 \mathrm{mg}$ once daily (od) for $10 \pm 4$ days is non-inferior to s.c. enoxaparin ( $40 \mathrm{mg}$ od) for $10 \pm 4$ days for the prevention of VTE in patients aged 40 years or older and hospitalized for a medical illness; secondly, to determine whether oral rivaroxaban $10 \mathrm{mg}$ od for $35 \pm 4$ days is superior to s.c. enoxaparin $40 \mathrm{mg}$ od for $10 \pm 4$ days (placebo continued until day $35 \pm 4$ ), in the same patient population. The principal safety objective is to compare the safety of rivaroxaban administered for $35 \pm 4$ days with enoxaparin administered for $10 \pm 4$ days.

\section{Methods}

Study design

MAGELLAN is a prospective, randomized, blinded (patient, care-giver, data-collector, and outcome-assessor), doubledummy, active comparator-controlled, multicenter, multinational clinical trial in acutely ill medical patients. The trial is being conducted in accordance with the Declaration of Helsinki and local regulations. Approval of the protocol was obtained from the appropriate ethics committee or institutional review board for all participating centers.

\section{Setting}

As of July 2010, 8,101 patients have been randomized in 556 centers from 52 countries (Table 1). 
Table 1 Number of sites enrolled by participating countries

\begin{tabular}{|c|c|}
\hline Geographic region and countries & $\begin{array}{l}\text { Number of } \\
\text { sites enrolled }\end{array}$ \\
\hline Africa: total & 14 \\
\hline South Africa & 14 \\
\hline Asia: total & 121 \\
\hline China & 43 \\
\hline Hong Kong & 2 \\
\hline India & 14 \\
\hline Indonesia & 3 \\
\hline Japan & 32 \\
\hline Korea & 7 \\
\hline Malaysia & 3 \\
\hline Pakistan & 3 \\
\hline Singapore & 6 \\
\hline Taiwan & 5 \\
\hline Thailand & 3 \\
\hline Australia and New Zealand: total & 14 \\
\hline Australia & 11 \\
\hline New Zealand & 3 \\
\hline Europe: total & 278 \\
\hline Austria & 14 \\
\hline Belgium & 10 \\
\hline Bulgaria & 8 \\
\hline Croatia & 6 \\
\hline Czech Republic & 7 \\
\hline Denmark & 5 \\
\hline Estonia & 4 \\
\hline Finland & 2 \\
\hline France & 22 \\
\hline Germany & 27 \\
\hline Greece & 10 \\
\hline Hungary & 8 \\
\hline Israel & 10 \\
\hline Italy & 21 \\
\hline Latvia & 6 \\
\hline Lithuania & 10 \\
\hline Luxembourg & 2 \\
\hline The Netherlands & 4 \\
\hline Norway & 4 \\
\hline Poland & 14 \\
\hline Portugal & 10 \\
\hline Russia & 8 \\
\hline Slovakia & 5 \\
\hline Slovenia & 6 \\
\hline Spain & 11 \\
\hline Sweden & 9 \\
\hline Switzerland & 6 \\
\hline Turkey & 6 \\
\hline Ukraine & 16 \\
\hline
\end{tabular}

Table 1 continued

\begin{tabular}{lc}
\hline Geographic region and countries & $\begin{array}{l}\text { Number of } \\
\text { sites enrolled }\end{array}$ \\
\hline United Kingdom & 7 \\
North America: total & 97 \\
Canada & 13 \\
Mexico & 12 \\
United States & 72 \\
South America: total & 32 \\
Argentina & 7 \\
Brazil & 8 \\
Chile & 2 \\
Colombia & 8 \\
Peru & 7 \\
\hline
\end{tabular}

\section{Patients}

Subjects are considered for enrollment if they are hospitalized for a specified acute medical illness, have a risk of VTE (reduced mobility with conditions as specified in inclusion criteria; Table 2), and their anticipated survival time is more than 6 months. Patients with acute infectious, inflammatory, or rheumatic diseases, and those with acute respiratory insufficiency, also require one or more wellcharacterized risk factors for VTE, such as a history of VTE or cancer [16, 24, 25]. All patients are required to give written informed consent. Patients are excluded from the study if they have conditions that contraindicate the use of enoxaparin or rivaroxaban. Patients are also excluded if they have certain concomitant conditions or diseases, including conditions that may increase the risk of bleeding, or if they require certain drugs or procedures (examples listed in Table 2).

\section{Treatment regimens}

Patients meeting the entry criteria are stratified by center using permuted blocks (size 4) and randomized using an interactive voice response system to allocate the blinded study medication. Patients are randomized to receive either s.c. enoxaparin $40 \mathrm{mg}$ od for $10 \pm 4$ days and oral rivaroxaban placebo for $35 \pm 4$ days, or oral rivaroxaban $10 \mathrm{mg}$ od for $35 \pm 4$ days and s.c. enoxaparin placebo for $10 \pm 4$ days. The active treatment period for the enoxaparin arm is from day 1 to day $10 \pm 4$ and for the rivaroxaban arm is from day 1 to day $35 \pm 4$ (day 1 is defined as the day of randomization). Patient follow-up continues until day $90 \pm 7$ (Fig. 1). 
Table 2 Principal inclusion and exclusion criteria for MAGELLAN

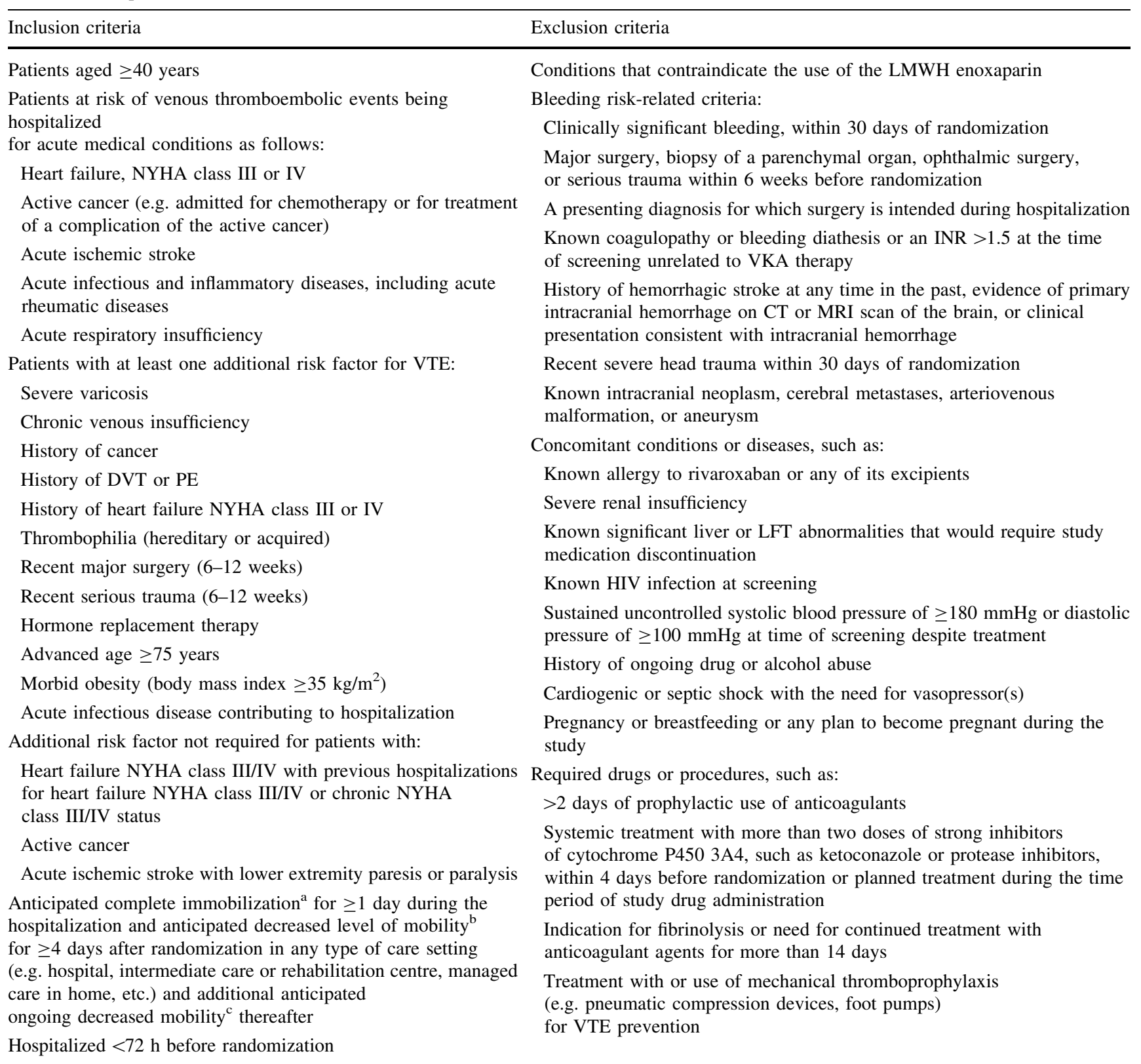

$C T$ computed tomography, DVT deep vein thrombosis, $H I V$ human immunodeficiency virus, INR international normalized ratio, $L F T$ liver function test, $L M W H$ low-molecular-weight-heparin, $M R I$ magnetic resonance imaging, NYHA New York Heart Association, $P E$ pulmonary embolism, VKA vitamin $\mathrm{K}$ antagonist, VTE venous thromboembolism

${ }^{a}$ Complete immobilization: the patient is totally confined by his or her illness to bed or chair. The patient may be allowed to use a bedside commode or with assistance may be allowed bathroom privileges

${ }^{b}$ Decreased mobility: immobilization caused by the illness requiring the patient to remain in bed or chair more than $50 \%$ of the time during daytime hours

${ }^{c}$ Ongoing decreased mobility: immobilization caused by the illness requiring the patient to remain in bed or chair during daytime hours more than was normal and usual for the patient prior to hospitalization 


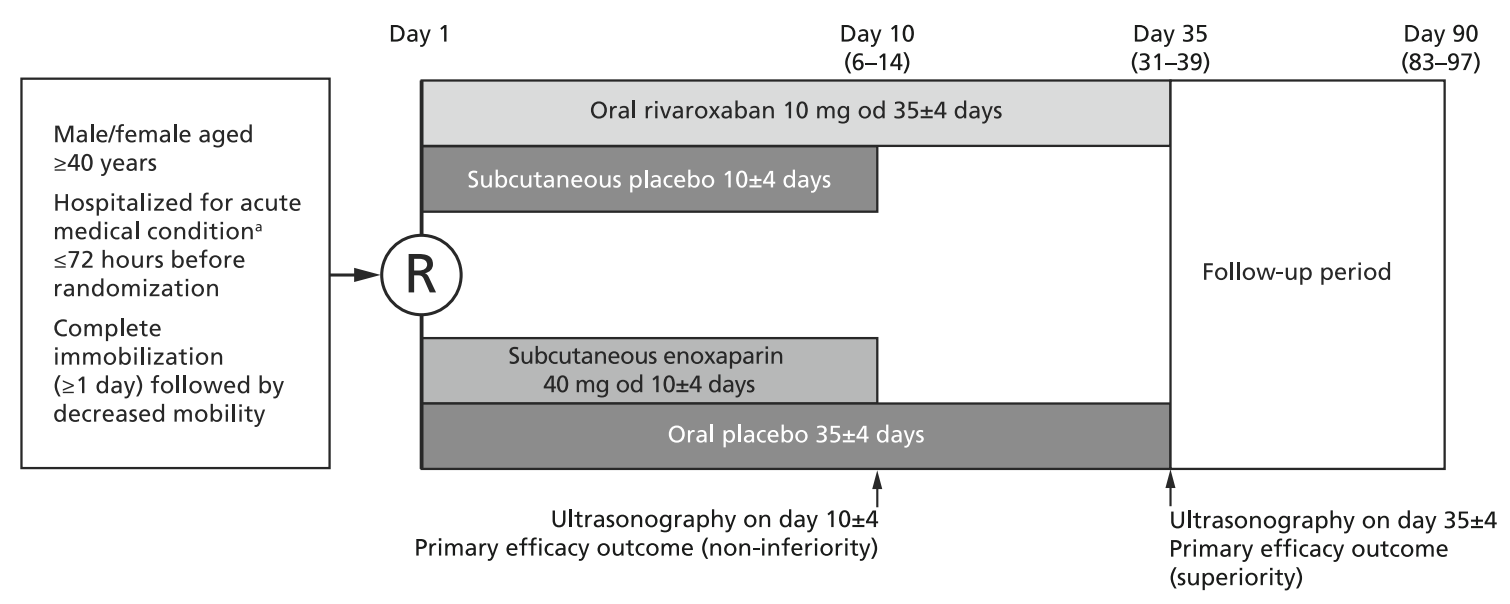

Fig. 1 MAGELLAN study design. ${ }^{\text {a }}$ Heart failure (New York Heart Association class III or IV), active cancer, acute ischemic stroke, acute infectious, acute inflammatory diseases or acute rheumatic diseases, or acute respiratory insufficiency. od once daily

\section{Study procedures}

Hospital unit and ward characteristics are recorded. During the screening period, a medical history is obtained. A physical examination, an electrocardiogram, a pregnancy test for women of childbearing potential and blood sampling for clinical chemistry (electrolytes, renal and liver function, and NT-proBNP), hematology (complete blood count), and coagulation (prothrombin time, prothrombinase-induced clotting time [ $\left[\mathrm{PiCT}^{\circledR}\right.$; Pentapharm, Basel, Switzerland], and D-dimer) are also performed. During the baseline (day 1 visit), rivaroxaban and matching placebo are administered orally with or without food; enoxaparin and matching placebo are administered subcutaneously.

For those subjects who give informed consent for the pharmacogenetic part of the study, a blood sample for pharmacogenetic analysis is taken (a retention sample is also taken). For subjects enrolled at selected centers, and willing to participate in the full PK/PD profile part of the study, blood samples are drawn before the first dose of study medication and at 1, 2, 3, 4, 6, 9, and $12 \mathrm{~h}$ post-dose.

Suspected cases of DVT are evaluated by bilateral lower extremity venous ultrasonography or other vascular imaging procedures of the lower extremities, e.g. venography. Suspected cases of PE are evaluated by thoracic spiral computed tomography, a ventilation perfusion lung scan with chest X-ray, or pulmonary angiography. If the diagnosis of DVT or PE is not confirmed, subjects continue to receive study medication. Mandatory routine bilateral lower extremity venous ultrasonography is performed after the last dose of study medication or matching placebo on day $10 \pm 4$ and on day $35 \pm 4$, within $24 \mathrm{~h}$ of their administration. In subjects prematurely discontinuing their treatment, bilateral ultrasonography is performed at that time and at day $35 \pm 4$. Patients are assessed on day $90 \pm 7$ for adverse events, symptomatic VTE, bleeding events, acute myocardial infarction, acute ischemic stroke, and death. Clinical chemistry is also performed for liver function testing. Symptoms of DVT or PE occurring during follow-up are verified by appropriate diagnostic testing as described above.

Efficacy and safety outcomes

There are two primary efficacy outcomes: the incidence of the composite of asymptomatic proximal DVT, symptomatic DVT (proximal or distal), symptomatic non-fatal PE, and VTE-related death from day 1 to day $10+4$ (test for non-inferiority); and the incidence of this composite outcome from day 1 to day $35+4$ (test for superiority).

The secondary efficacy outcomes are the incidence of the composite of asymptomatic proximal DVT, symptomatic DVT, symptomatic non-fatal PE, and all-cause mortality up to day $10+4$ and day $35+4$ (major secondary outcome); symptomatic VTE up to day $10+4$, day $35+4$, and day $90+7$; and all-cause mortality up to day $90+7$. Net clinical benefit will be assessed by the composite outcome of asymptomatic proximal DVT, symptomatic DVT, symptomatic non-fatal PE, VTE-related death, treatmentemergent major bleeding, and non-major clinically relevant bleeding up to day $10+4$ and day $35+4$. The incidence of each component of the primary efficacy outcomes and the composite of cardiovascular death, acute myocardial infarction, or acute ischemic stroke up to day $10+4$, day $35+4$, and day $90+7$ are also secondary outcomes.

The primary safety outcome is the incidence of the composite of treatment-emergent major bleeding and nonmajor clinically relevant bleeding observed not later than 2 days after the last intake of study medication. Bleeding events are defined in Table 3. Bleeding events observed more than 2 days after the last intake of study medication will be considered separately. Minimal bleeding events, 
Table 3 Definitions of bleeding events

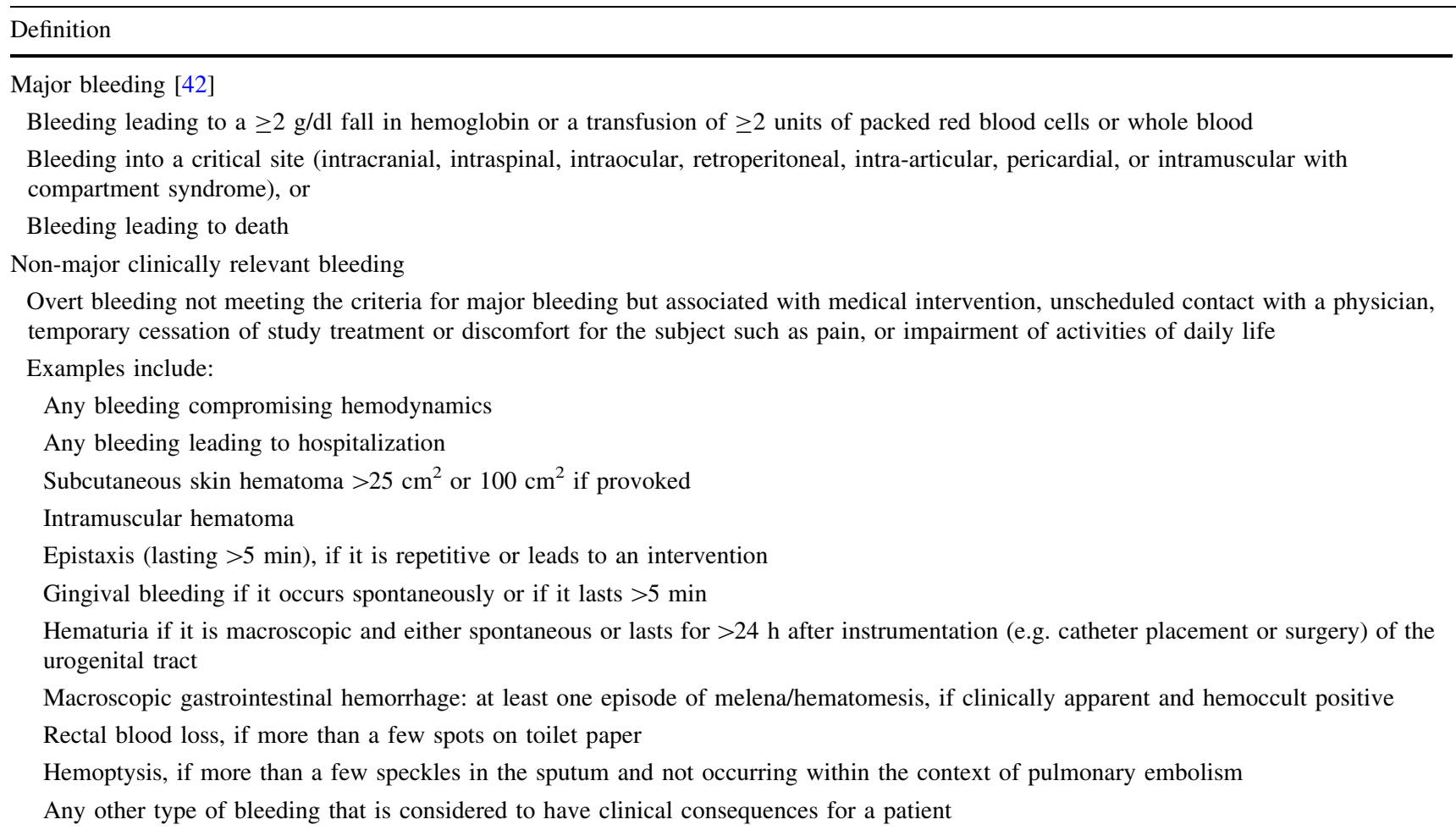

i.e. any other overt bleeding episodes not meeting the criteria for major bleeding or non-major clinically relevant bleeding events, will also be summarized. Other safety outcomes include treatment-emergent adverse events and abnormal laboratory parameters.

\section{PK and PD parameters}

A target of 125 patients willing to participate in a full PK/PD profile will be stratified into five groups of 25 patients for the major diagnostic groups.

Based on the plasma concentration-time data, PK parameters of rivaroxaban will be calculated on day 1 and day $10 \pm 4$ for the data-rich profile obtained in this subset of patients. These parameters include area under the curve, maximum concentration of drug in plasma, elimination half-life, and apparent clearance.

\section{Pharmacogenetics}

The pharmacogenetic assessment allows analysis of genes involved in the absorption, distribution, metabolism, and excretion of the study drug. Genes involved in coagulation, thrombotic diseases, treatment response, and safety could also be evaluated. No prospective analyses are planned for the pharmacogenetic samples in the study, and samples will be stored and analyzed only if other data collected in the study result in a hypothesis that can be tested by genetic analysis.

Health economics outcome research

Healthcare resource utilization is evaluated and includes primary hospitalization type and duration, re-hospitalization and emergency room visits, healthcare professional visits, outpatient surgery and consultations, and need for inpatient or outpatient rehabilitation or skilled nursing facility stay. Quality of life measures (EQ-5D [European Quality of Life] questionnaire) and patient-reported outcomes (SF-12 [Short Form] survey) are also being collected.

Statistical analyses and sample size

A statistical analysis plan has been completed. Subjects are valid for the safety analysis if at least one dose of study medication is administered. Two efficacy analysis populations are defined, both for the non-inferiority analysis and for the superiority analysis. In both populations, patients are eligible for the modified intention-to-treat (mITT) analysis if they are valid for the safety analysis and have an adequate assessment of VTE, defined as an adequate assessment of DVT or PE, if applicable. Patients are eligible for the per-protocol analysis if they are valid for the mITT analysis, have an adequate assessment of VTE 
performed not later than 2 days after the last intake of study drug, meet the inclusion criteria, and have no major protocol violations.

Sensitivity analyses will be performed for the primary efficacy endpoint considering all randomized subjects. A method described for the analysis of a binary composite endpoint with missing data in components will be used [26]. The two components of the binary composite endpoint will be the assessment of an asymptomatic proximal DVT from the bilateral lower extremity venous ultrasonography and the assessment of symptomatic lower extremity DVT (proximal or distal), symptomatic non-fatal PE, or VTE-related death. The latter assessment will be considered to have been evaluated in all subjects because it is assumed that such an event would have been reported if it occurred. The former assessment will be considered to have been evaluated in all subjects with evaluable ultrasonography (within the assessment period). This approach derives the probabilities of the outcomes and constructs the overall rate based on the estimated probabilities of the two components. A 95\% asymptotic (unstratified) confidence interval around the relative risk will be calculated.

\section{Efficacy analysis}

All statistical tests will be one-sided with a type I error rate of $2.5 \%$; two-sided $95 \%$ CIs will be given. A multiple testing approach will be employed to evaluate the two primary efficacy outcomes [27]. If the test for non-inferiority at day $10+4$ is met, a test for superiority will be applied using the method described for the primary efficacy outcomes. If both primary outcomes are significant at the one-sided level $(2.5 \%)$ then both outcomes can be declared statistically significant. If one $P$-value is greater than $2.5 \%$, the $P$-value for the other primary outcome must be less than $1.25 \%$ before significance for this outcome can be declared.

For both primary efficacy outcomes, the relative risk ratio of the incidence rates (rivaroxaban/enoxaparin) will be estimated based on a stratified Mantel-Haenszel model (stratified by geographic region). The primary analysis population for superiority will be the mITT population. The primary analysis population for non-inferiority will be the per-protocol population.

The secondary outcomes of the composite of asymptomatic proximal DVT, symptomatic DVT, symptomatic non-fatal PE, and all-cause mortality up to day $35+4$, and adverse events will be analyzed in the same way as the primary outcomes. The outcomes of symptomatic VTE and death will be analyzed in the safety population; the hazard ratios (rivaroxaban/enoxaparin) will be estimated based on a stratified proportional hazards model (stratified by geographic region).
Safety analysis

All statistical tests will be two-sided with a type I error rate of 5\%; two-sided $95 \%$ CIs will be given. The incidence of the composite of major bleeding and non-major clinically relevant bleeding and the incidence of major bleeding will be analyzed as described for the primary efficacy outcomes. All other safety data will be analyzed descriptively.

No interim analysis was planned after the first amendment to the protocol. The introduction of the mandatory lower extremity vascular ultrasonography on day $10 \pm 4$ mitigated the need for an interim analysis because of a safety concern because all patients with proximal asymptomatic leg thrombosis would be identified and treated as required if detected during the day $10 \pm 4$ period.

\section{Exploratory analyses}

The incidence rates of the primary efficacy endpoints and the major secondary endpoint in each treatment group as well as the relative risk and the corresponding non-stratified exact two-sided $95 \%$ CIs will additionally be assessed by geographic region and country as well as by demographic variables and other covariates.

\section{Determination of sample size}

The determination of sample size takes into account the assumptions for both primary efficacy outcomes and allows for a joint power of at least $90 \%$. A sample size of 2,876 valid patients per group was estimated based on the following assumptions: a $2.2 \%$ event rate in the control group at day $10+4$ [17-19], a relative reduction of at least $35 \%$ in event rate with rivaroxaban at day $10+4$, a $4 \%$ event rate in the control group at day $35+4[17-19,28]$, a relative reduction of at least $40 \%$ in event rate with rivaroxaban at day $35+4$, and a joint probability of a type II error of less than $10 \%$. Assuming an invalidity rate of up to $20-30 \%$, the total sample size of randomized subjects is 7,190-8,220. The sample size estimate assumes a noninferiority margin of 1.5 based on the lower $95 \%$ confidence bound for the relative risk of placebo versus active treatment in a meta-analysis of three placebo-controlled trials in acutely ill medical patients [12].

\section{$P K$ and $P D$}

Descriptive statistics will be calculated for plasma concentration parameters. Exploratory analyses will be performed to relate coagulation parameters to bleeding events and to relate coagulation parameters to venous thromboembolic events. 
Ultrasonography procedures

To assess asymptomatic proximal DVT, bilateral ultrasonography of the proximal veins of the lower extremity is performed in all subjects. Veins identified are the common femoral vein, the deep femoral vein, the superficial femoral vein, the popliteal vein, and the trifurcation of the calf veins.

The lack of compressibility of a vein under probe pressure is considered to be the primary criterion for the demonstration of thrombosis. Compression maneuvers with the ultrasonography transducer in the transverse plane are performed at $2-3 \mathrm{~cm}$ intervals along the veins studied. The ultrasonography examination is adequate if all vein segments are documented by appropriately annotated continuous recordings or still images, and allows the assessment of compressibility for each of the required veins. An Ultrasonography Adjudication Committee (UAC) adjudicates all scheduled and unscheduled ultrasonographies performed during the treatment period in a blinded manner. The adjudication of the UAC is the basis for the final primary efficacy analysis.

Clinical events procedures

A Clinical Events Adjudication Committee centrally adjudicates and/or classifies the primary and secondary efficacy and safety (all bleeding) clinical events.

\section{Protocol amendments}

Modifications to the study protocol were only implemented with agreement from both the sponsor and the Steering Committee.

After discussion with the health authorities, the following changes were made to the study protocol via its first amendment. Patients with diabetes mellitus or patients who would fall into the broad category of having 'other' medical illnesses were excluded. For patients with acute infectious, inflammatory and rheumatic diseases, and acute respiratory insufficiency, one or more additional risk factors for VTE were stipulated as a requirement for inclusion in the study. In addition, the required duration of immobilization was extended to beyond the 4 days of hospitalization, and a second routine lower extremity venous ultrasonography was added to patient assessment, performed at the time of parenteral study medication discontinuation (day 10). This efficacy outcome was to test for non-inferiority of rivaroxaban compared with enoxaparin for efficacy.

Further amendments included the addition of acute infectious disease contributing to hospitalization as an inclusion criterion and increasing the period of hospitalization before randomization from 48 to $72 \mathrm{~h}$. Hospital stay
Table 4 Primary diagnosis of subjects in the MAGELLAN study safety population

\begin{tabular}{lr}
\hline Subject characteristics & \\
\hline Number of subjects in the safety population & 7998 \\
Primary diagnosis $(\%)^{\mathrm{a}}$ & \\
Acute infectious and inflammatory disease & 47.3 \\
Acute heart failure & 32.4 \\
Acute respiratory insufficiency & 28.0 \\
Acute stroke & 17.3 \\
Active cancer & 7.3 \\
Other diseases & 0.7 \\
\hline
\end{tabular}

${ }^{a}$ Patients could have more than one primary diagnosis

and duration vary widely between countries for patients with identical admitting diagnoses and the venue of immobilization required for inclusion of a patient was expanded to any type of healthcare setting.

\section{Current status of the study}

As of July 2010, 8,101 patients were included in the study and enrollment was stopped. The last patient visits took place in October 2010.

The patients cover a broad age range (40-105 years) and have a diverse range of primary diagnoses (Table 4).

\section{Discussion}

MAGELLAN will be the first randomized clinical trial of VTE prevention in acutely ill medical patients to directly compare rivaroxaban, a new generation oral anticoagulant, with s.c. enoxaparin administered for $10 \pm 4$ days. The active comparator phase of this study will allow an unbiased comparison of rivaroxaban with enoxaparin and will also provide insight into the potential benefits of oral compared with s.c. thromboprophylaxis administration.

MAGELLAN will also assess the efficacy and safety of extended-duration thromboprophylaxis with rivaroxaban. There is strong evidence for the benefits of extendedduration thromboprophylaxis in elective abdominal cancer surgery $[29,30]$ and in hip replacement [31-33] and fracture surgery [34]. The use of extended-duration LMWH thromboprophylaxis up to 35 days is well established in these surgeries. Recently, oral rivaroxaban $10 \mathrm{mg}$ od administered for 35 days was shown to significantly reduce the incidence of VTE in patients undergoing total hip replacement surgery compared with s.c. enoxaparin $40 \mathrm{mg}$ od administered for 35 days [5].

The rivaroxaban dose selected for use in the MAGELLAN study was based on phase II studies in orthopedic surgery, 
which demonstrated rivaroxaban $10 \mathrm{mg}$ od had comparable efficacy and safety to enoxaparin $40 \mathrm{mg}$ od; phase III studies confirmed this $[5,6,8,35,36]$. The enoxaparin regimen chosen for the comparator group is approved for use in all participating countries [37].

In the current study, asymptomatic VTE is diagnosed using a standardized ultrasonography protocol, as first utilized in the PREVENT study [18]. The advantages of ultrasonography compared with venography are that it is convenient, readily available, non-invasive, repeatable, and does not expose patients to ionizing radiation or contrast media. Standardized ultrasonography techniques limit the sensitivity issues previously associated with ultrasonography [38]. However, a consensus on standardization of venous ultrasound as an endpoint outcome is still needed [39].

Risk assessment of acutely ill medical patients is important to allow physicians to prescribe appropriate anticoagulant therapy. In clinical trials of thromboprophylaxis in acutely ill medical patients, variation exists between the VTE risk factors used as patient inclusion criteria. When the MAGELLAN study protocol was designed, careful consideration was given to which VTE risk-factor-related inclusion criteria were most appropriate. Acute medical illnesses and predisposing risk factors (e.g. age or previous VTE) were selected based on published randomized controlled trials and other types of studies.

This study, which is recruiting from 52 countries and includes centers in Asia, Europe, North America, and Africa, will provide a unique opportunity to evaluate the risk of VTE and the potential benefits of extended-duration thromboprophylaxis in a truly multinational patient population.

Other than the EXCLAIM study [40], MAGELLAN is the only study in this patient population (completed or ongoing) to include patients with active cancer and acute ischemic stroke - conditions associated with a high risk of VTE $[12,13]$. Cancer in particular is a major risk factor, with up to one in five diagnosed cases of VTE occurring in a person with cancer [41]. Because of the large number of patients in this study, MAGELLAN will also provide data on VTE epidemiology in subgroups of patients where there is little data, i.e. patients with acute infection or heart failure.

MAGELLAN will provide further evidence as to whether extended-duration therapy is more effective than standard-duration therapy in a diverse population of acutely ill medical patients. Furthermore, it will provide information on the efficacy, safety, and pharmacologic profile of the oral anticoagulant rivaroxaban in this patient population, as well as health economics outcome research data. Extended-duration therapy with a well-tolerated and effective oral anticoagulant given once daily has the potential to simplify thromboprophylaxis in acutely ill medical patients, particularly during the post-hospitaldischarge period. This could improve patient compliance and physician adherence to guidelines, ultimately reducing VTE-associated morbidity and mortality.

\section{Addendum}

The protocol amendments described were implemented in response to regulatory authority requests, to comply with local regulations and requirements, or to comply with labeling requirements.

Acknowledgments This study was supported by Bayer Schering Pharma AG and Johnson \& Johnson Pharmaceutical Research \& Development, L.L.C. The authors would like to acknowledge: Rong Chen MD PhD, Medical Expert Bayer HealthCare; Isabelle Meng MD PhD, Medical Expert Bayer HealthCare; Linda Li MD PhD, Medical Expert Bayer HealthCare; Sonja Dalle-Ave MD, Medical Expert Bayer HealthCare; Eva Muehlhofer MD, Medical Expert Bayer HealthCare; Horst Beckmann PhD, Statistician Bayer HealthCare; Alice Benson MS, Statistician Bayer HealthCare; Andrea Dusczyscyn, Study Manager Bayer HealthCare; Lynda Fielding, Study Manager Bayer HealthCare; Teresa Twomey, Study Manager Bayer HealthCare. The authors would also like to acknowledge Sarah Atkinson who provided editorial support with funding from Bayer Schering Pharma AG and Johnson \& Johnson Pharmaceutical Research \& Development, L.L.C.

Financial support This analysis was supported by Bayer Schering Pharma AG and Johnson \& Johnson Pharmaceutical Research \& Development, L.L.C.

Open Access This article is distributed under the terms of the Creative Commons Attribution Noncommercial License which permits any noncommercial use, distribution, and reproduction in any medium, provided the original author(s) and source are credited.

\section{References}

1. Alikhan R, Cohen AT (2009) Heparin for the prevention of venous thromboembolism in general medical patients (excluding stroke and myocardial infarction). Cochrane Database Syst Rev CD003747

2. Dentali F, Douketis JD, Gianni M, Lim W, Crowther MA (2007) Meta-analysis: anticoagulant prophylaxis to prevent symptomatic venous thromboembolism in hospitalized medical patients. Ann Intern Med 146:278-288

3. Collins R, Scrimgeour A, Yusuf S, Peto R (1988) Reduction in fatal pulmonary embolism and venous thrombosis by perioperative administration of subcutaneous heparin. Overview of results of randomized trials in general, orthopedic, and urologic surgery. N Engl J Med 318:1162-1173

4. Geerts WH, Bergqvist D, Pineo GF et al (2008) Prevention of venous thromboembolism: American College of Chest Physicians evidence-based clinical practice guidelines (8th edition). Chest $133: 381 \mathrm{~S}-453 \mathrm{~S}$ 
5. Eriksson BI, Borris LC, Friedman RJ et al (2008) Rivaroxaban versus enoxaparin for thromboprophylaxis after hip arthroplasty. N Engl J Med 358:2765-2775

6. Kakkar AK, Brenner B, Dahl OE et al (2008) Extended duration rivaroxaban versus short-term enoxaparin for the prevention of venous thromboembolism after total hip arthroplasty: a doubleblind, randomised controlled trial. Lancet 372:31-39

7. Lassen MR, Raskob GE, Gallus A et al (2010) Apixaban versus enoxaparin for thromboprophylaxis after knee replacement (ADVANCE-2): a randomised double-blind trial. Lancet 375: 807-815

8. Lassen MR, Ageno W, Borris LC et al (2008) Rivaroxaban versus enoxaparin for thromboprophylaxis after total knee arthroplasty. N Engl J Med 358:2776-2786

9. Turpie AGG, Lassen MR, Davidson BL et al (2009) Rivaroxaban versus enoxaparin for thromboprophylaxis after total knee arthroplasty (RECORD4): a randomised trial. Lancet 373:1673-1680

10. NICE (2010) Venous thromboembolism: reducing the risk of venous thromboembolism (deep vein thrombosis and pulmonary embolism) in patients admitted to hospital. http://www.nice.org. uk/nicemedia/pdf/CG92FullGuideline.pdf. Accessed 6 July 2010

11. Ageno W, Turpie AGG (2002) Deep venous thrombosis in the medically ill. Curr Hematol Rep 1:73-78

12. Leizorovicz A, Mismetti P (2004) Preventing venous thromboembolism in medical patients. Circulation 110:IV13-IV19

13. Cohen AT, Alikhan R, Arcelus JI et al (2005) Assessment of venous thromboembolism risk and the benefits of thromboprophylaxis in medical patients. Thromb Haemost 94:750-759

14. Samama MM (2000) An epidemiologic study of risk factors for deep vein thrombosis in medical outpatients: the Sirius study. Arch Intern Med 160:3415-3420

15. Heit JA, Silverstein MD, Mohr DN et al (2001) The epidemiology of venous thromboembolism in the community. Thromb Haemost 86:452-463

16. Heit JA, O'Fallon WM, Petterson TM et al (2002) Relative impact of risk factors for deep vein thrombosis and pulmonary embolism: a population-based study. Arch Intern Med 162:1245-1248

17. Samama MM, Cohen AT, Darmon JY et al (1999) A comparison of enoxaparin with placebo for the prevention of venous thromboembolism in acutely ill medical patients. Prophylaxis in Medical Patients with Enoxaparin Study Group. N Engl J Med 341:793-800

18. Leizorovicz A, Cohen AT, Turpie AGG, Olsson CG, Vaitkus PT, Goldhaber SZ (2004) Randomized, placebo-controlled trial of dalteparin for the prevention of venous thromboembolism in acutely ill medical patients. Circulation 110:874-879

19. Cohen AT, Davidson BL, Gallus AS et al (2006) Efficacy and safety of fondaparinux for the prevention of venous thromboembolism in older acute medical patients: randomised placebo controlled trial. BMJ 332:325-329

20. Cohen AT, Tapson VF, Bergmann JF et al (2008) Venous thromboembolism risk and prophylaxis in the acute hospital care setting (ENDORSE study): a multinational cross-sectional study. Lancet 371:387-394

21. Tapson VF, Decousus H, Pini M et al (2007) Venous thromboembolism prophylaxis in acutely ill hospitalized medical patients: findings from the International Medical Prevention Registry on Venous Thromboembolism. Chest 132:936-945

22. Hull RD, Schellong SM, Tapson VF et al (2010) Extendedduration venous thromboembolism prophylaxis in acutely ill medical patients with recently reduced mobility: a randomized trial. Ann Intern Med 153:8-18

23. Perzborn E, Roehrig S, Straub A, Kubitza D, Mueck W, Laux V (2010) Rivaroxaban: a new oral factor Xa inhibitor. Arterioscler Thromb Vasc Biol 30:376-381

24. White RH (2003) The epidemiology of venous thromboembolism. Circulation 107:I4-I8
25. Alikhan R, Cohen AT, Combe S et al (2004) Risk factors for venous thromboembolism in hospitalized patients with acute medical illness: analysis of the MEDENOX study. Arch Intern Med 164:963-968

26. Quan H, Zhang D, Zhang J, Devlamynck L (2007) Analysis of a binary composite endpoint with missing data in components. Stat Med 26:4703-4718

27. Hochberg Y, Hommel G (1998) Simes' test of multiple hypotheses. In: Kotz S, Read CB, Banks DL (eds) Encyclopedia of statistical sciences - update series. Wiley, New York, pp 418-422

28. Hull R, Schellong S, Tapson V et al (2007) Extended-duration venous thromboembolism (VTE) prophylaxis in acutely ill medical patients with recent reduced mobility: the EXCLAIM study. J Thromb Haemost 5(suppl 2) [abstract O-S-001]

29. Bergqvist D, Agnelli G, Cohen AT et al (2002) Duration of prophylaxis against venous thromboembolism with enoxaparin after surgery for cancer. N Engl J Med 346:975-980

30. Rasmussen MS, Jorgensen LN, Wille-Jørgensen P et al (2006) Prolonged prophylaxis with dalteparin to prevent late thromboembolic complications in patients undergoing major abdominal surgery: a multicenter randomized open-label study. J Thromb Haemost 4:2384-2390

31. Hull RD, Pineo GF (1999) Extended prophylaxis against venous thromboembolism following total hip and knee replacement. Haemostasis 29:23-31

32. Eikelboom JW, Quinlan DJ, Douketis JD (2001) Extendedduration prophylaxis against venous thromboembolism after total hip or knee replacement: a meta-analysis of the randomised trials. Lancet 358:9-15

33. Hull RD, Pineo GF, Stein PD et al (2001) Extended out-ofhospital low-molecular-weight heparin prophylaxis against deep venous thrombosis in patients after elective hip arthroplasty: a systematic review. Ann Intern Med 135:858-869

34. Eriksson BI, Lassen MR (2003) Duration of prophylaxis against venous thromboembolism with fondaparinux after hip fracture surgery: a multicenter, randomized, placebo-controlled, doubleblind study. Arch Intern Med 163:1337-1342

35. Eriksson BI, Borris L, Dahl OE et al (2006) Oral, direct Factor Xa inhibition with BAY 59-7939 for the prevention of venous thromboembolism after total hip replacement. J Thromb Haemost 4:121-128

36. Eriksson BI, Borris LC, Dahl OE et al (2006) A once-daily, oral, direct Factor Xa inhibitor, rivaroxaban (BAY 59-7939), for thromboprophylaxis after total hip replacement. Circulation 114:2374-2381

37. Sanofi-Aventis (2009) Prescribing information. http://products. sanofi-aventis.us/lovenox/lovenox.pdf. Accessed 19 Oct 2010

38. Schellong SM, Beyer J, Kakkar AK et al (2007) Ultrasound screening for asymptomatic deep vein thrombosis after major orthopaedic surgery: the VENUS study. J Thromb Haemost 5:1431-1437

39. Schellong SM (2008) Venous ultrasonography in symptomatic and asymptomatic patients: an updated review. Curr Opin Pulm Med 14:374-380

40. Hull RD, Schellong SM, Tapson VF et al (2006) Extendedduration thromboprophylaxis in acutely ill medical patients with recent reduced mobility: methodology for the EXCLAIM study. J Thromb Thrombolysis 22:31-38

41. Lyman GH, Khorana AA, Falanga A et al (2007) American Society of Clinical Oncology guideline: recommendations for venous thromboembolism prophylaxis and treatment in patients with cancer. J Clin Oncol 25:5490-5505

42. Schulman S, Kearon C (2005) Definition of major bleeding in clinical investigations of antihemostatic medicinal products in non-surgical patients. J Thromb Haemost 3:692-694 